This is a self-archived version of an original article. This version may differ from the original in pagination and typographic details.

Author(s): Kumar, Priyank V.; Rossi, Tuomas P.; Marti-Dafcik, Daniel; Reichmuth, Daniel;

Title: Plasmon-Induced Direct Hot-Carrier Transfer at Metal-Acceptor Interfaces

Year: 2019

Version: Accepted version (Final draft)

Copyright: (c) 2019 American Chemical Society.

Rights: In Copyright

Rights url: http://rightsstatements.org/page//nC/1.0/?language=en

Please cite the original version:

Kumar, P. V., Rossi, T. P., Marti-Dafcik, D., Reichmuth, D., Kuisma, M., Erhart, P., Puska, M. J., \& Norris, D. J. (2019). Plasmon-Induced Direct Hot-Carrier Transfer at Metal-Acceptor Interfaces. ACS Nano, 13(3), 3188-3195. https://doi.org/10.1021/acsnano.8b08703 
Subscriber access provided by JYVASKYLAN UNIV

\section{Article}

\section{Plasmon-Induced Direct Hot-Carrier Transfer at Metal-Acceptor Interfaces}

Priyank V. Kumar, Tuomas P. Rossi, Daniel M- Dafcik, Daniel Reichmuth, Mikael Kuisma, Paul Erhart, Martti J. Puska, and David J. Norris

ACS Nano, Just Accepted Manuscript • DOI: 10.1021/acsnano.8b08703 • Publication Date (Web): 15 Feb 2019

Downloaded from http://pubs.acs.org on February 18, 2019

\section{Just Accepted}

"Just Accepted" manuscripts have been peer-reviewed and accepted for publication. They are posted online prior to technical editing, formatting for publication and author proofing. The American Chemical Society provides "Just Accepted" as a service to the research community to expedite the dissemination of scientific material as soon as possible after acceptance. "Just Accepted" manuscripts appear in full in PDF format accompanied by an HTML abstract. "Just Accepted" manuscripts have been fully peer reviewed, but should not be considered the official version of record. They are citable by the Digital Object Identifier (DOI®). "Just Accepted" is an optional service offered to authors. Therefore, the "Just Accepted" Web site may not include all articles that will be published in the journal. After a manuscript is technically edited and formatted, it will be removed from the "Just Accepted" Web site and published as an ASAP article. Note that technical editing may introduce minor changes to the manuscript text and/or graphics which could affect content, and all legal disclaimers and ethical guidelines that apply to the journal pertain. ACS cannot be held responsible for errors or consequences arising from the use of information contained in these "Just Accepted" manuscripts. 


\title{
Plasmon-Induced Direct Hot-Carrier Transfer at Metal-Acceptor Interfaces
}

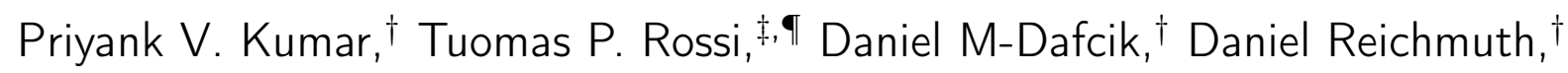 \\ Mikael Kuisma, ${ }^{\S}$ Paul Erhart, ${ }^{\ddagger}$ Martti J. Puska, a and David J. Norris*,† \\ $\dagger$ Optical Materials Engineering Laboratory, ETH Zurich, 8092 Zurich, Switzerland \\ $\ddagger$ Department of Physics, Chalmers University of Technology, 41296 Gothenburg, Sweden \\ IDepartment of Applied Physics, Aalto University, 00076 Aalto, Finland \\ $\S$ Department of Chemistry, Nanoscience Center, University of Jyväskylä, 40014 Jyväskylä, \\ Finland \\ E-mail: dnorris@ethz.ch
}




\begin{abstract}
Plasmon-induced hot-carrier transfer from a metal nanostructure to an acceptor is known to occur via two key mechanisms: (i) indirect transfer, where the hot carriers are produced in the metal nanostructure and subsequently transferred to the acceptor, and (ii) direct transfer, where the plasmons decay by directly exciting carriers from the metal to the acceptor. Unfortunately, an atomic-level understanding of the direct-transfer process, especially with regard to its quantification, remains elusive even though it is estimated to be more efficient compared to the indirect-transfer process. This is due to experimental challenges in separating direct from indirect transfer as both processes occur simultaneously at femtosecond timescales. Here, we employ timedependent density-functional theory simulations to isolate and study the direct-transfer process at a model metal-acceptor $\left(\mathrm{Ag}_{147}-\mathrm{Cd}_{33} \mathrm{Se}_{33}\right)$ interface. Our simulations show that, for a 10-femtosecond Gaussian laser pulse tuned to the plasmon frequency, the plasmon formed in the $\mathrm{Ag}_{147}-\mathrm{Cd}_{33} \mathrm{Se}_{33}$ system decays within $10 \mathrm{fs}$ and induces the direct transfer with a probability of about $40 \%$. We decompose the direct-transfer process further and demonstrate that the direct injection of both electrons and holes into the acceptor, termed direct hot-electron transfer (DHET) and direct hot-hole transfer (DHHT), take place with similar probabilities of about $20 \%$ each. Finally, effective strategies to control and tune the probabilities of DHET and DHHT processes are proposed. We envision our work to provide guidelines toward the design of metal-acceptor interfaces that enable more efficient plasmonic hot-carrier devices.
\end{abstract}

\title{
Keywords
}

plasmon decay, direct transfer, hot electrons, hot holes, time-dependent density-functional theory, probability 
The interaction of photons with a metallic nanoparticle can excite localized surface plasmons that subsequently decay non-radiatively to generate energetic charge carriers (referred to as hot electrons and hot holes). ${ }^{1,2}$ Since these hot carriers can be transferred to a chemically attached acceptor such as a semiconductor or a molecule, this process is potentially useful for technologies such as photovoltaics, ${ }^{3}$ photodetection, ${ }^{4,5}$ photon upconversion, ${ }^{6}$ and photocatalysis. $^{2,7,8}$ Hot-carrier transfer is commonly believed to occur indirectly (indirect transfer), where the hot carriers are first produced in the metal and then move to the acceptor (Figure 1a). ${ }^{1,2,9,10}$ Experiments ${ }^{11-14}$ and theoretical calculations ${ }^{15-23}$ that have characterized this mechanism have, however, pointed out that this pathway can be inefficient. Hot carriers generated in the metal can quickly relax back to the Fermi level via carrier-carrier scattering before being transferred to the acceptor.

Fortunately, recent experiments at metal-semiconductor ${ }^{24-27}$ and metal-molecule ${ }^{28,29}$ interfaces have provided strong evidence for another carrier-transfer mechanism that can operate simultaneously, termed direct transfer. This process is also referred to as coherent charge transfer, ${ }^{25,26}$ plasmon-induced interfacial charge-transfer transition when the acceptor is a semiconductor, ${ }^{24}$ and chemical interface damping in the case of adsorbed molecules. ${ }^{2,30-32}$ In this case, surface plasmons interact directly with empty acceptor states and dephase by directly injecting carriers into the acceptor (Figure 1b). This pathway can involve two transitions across the interface: (i) carriers in occupied states in the metal transfer to unoccupied states in the acceptor, generating electrons (holes) in the acceptor (metal), a process that we refer to as direct hot-electron transfer (DHET), and (ii) carriers in occupied acceptor states transfer to unoccupied states in the metal, generating holes (electrons) in the acceptor (metal), a process we refer to as direct hot-hole transfer (DHHT). These direct-transfer processes overcome the problem of fast relaxation of hot carriers in the indirect-transfer mechanism and present an opportunity to obtain more efficient plasmonic hot-carrier devices. ${ }^{10,30,31}$

Since direct transfer, however, occurs on an ultrafast timescale and is experimentally 
(a)

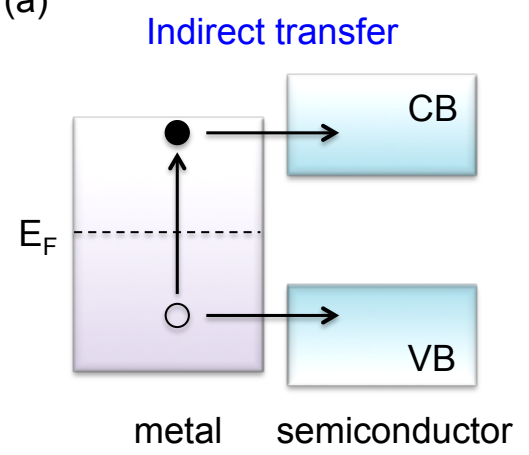

(b)

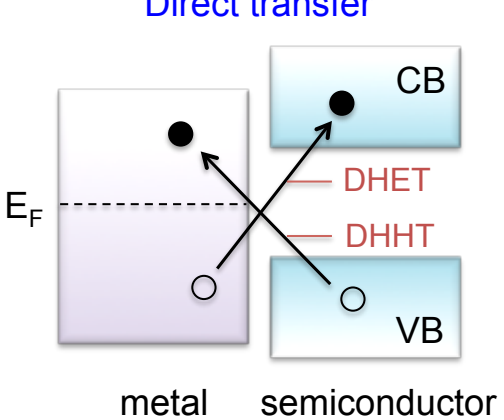

(c) Intra-acceptor excitation
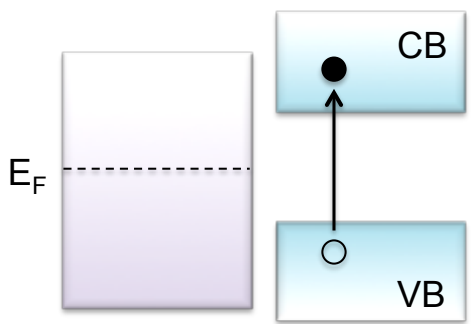

metal semiconductor

Figure 1: Schematic representation of (a) the indirect-transfer process, (b) the direct-transfer process, and (c) the intra-acceptor excitation at a metal-semiconductor interface (see text for more details). The two direct-transfer transitions, direct hot-electron transfer (DHET) and direct hot-hole transfer (DHHT), are also shown. $E_{\mathrm{F}}, \mathrm{CB}$ and VB represent Fermi level, conduction and valence band, respectively. Full and empty circles denote electrons and the holes, respectively. A similar scheme can be drawn for a metal-molecule interface.

difficult to distinguish from indirect transfer that operates in parallel, the direct-transfer mechanism remains poorly understood at the atomic scale. ${ }^{2,8,24-26}$ It has been particularly challenging to isolate direct-transfer transitions (DHET and DHHT), understand their characteristics, and quantify them accurately. While theoretical calculations could help in this direction, a clear methodology to address these issues rooted in a rigorous first-principles approach is missing.

Here, we present an ab initio approach based on real-time time-dependent densityfunctional theory ${ }^{33}$ (RT-TDDFT) to capture the direct-transfer process at a model metalacceptor interface formed by a metallic $\mathrm{Ag}_{147}$ nanoparticle and a semiconducting $\mathrm{Cd}_{33} \mathrm{Se}_{33}$ cluster (see Figure 2). The icosahedral $\mathrm{Ag}_{147}$ nanoparticle supports a strong plasmon and has been previously studied by some of the authors. ${ }^{34,35}$ The model semiconductor $\mathrm{Cd}_{33} \mathrm{Se}_{33}$ structure has been studied in Refs. 36 and 37.

Among other candidates, Ag/CdSe assemblies have been utilized as plasmonic photocatalysts, ${ }^{38}$ thus justifying their choice as a model system in our simulations. We demonstrate that the plasmon formed in the $\mathrm{Ag}_{147}-\mathrm{Cd}_{33} \mathrm{Se}_{33}$ system due to the application of a laser pulse decays within 10 fs and induces direct-transfer transitions across the interface with significant 

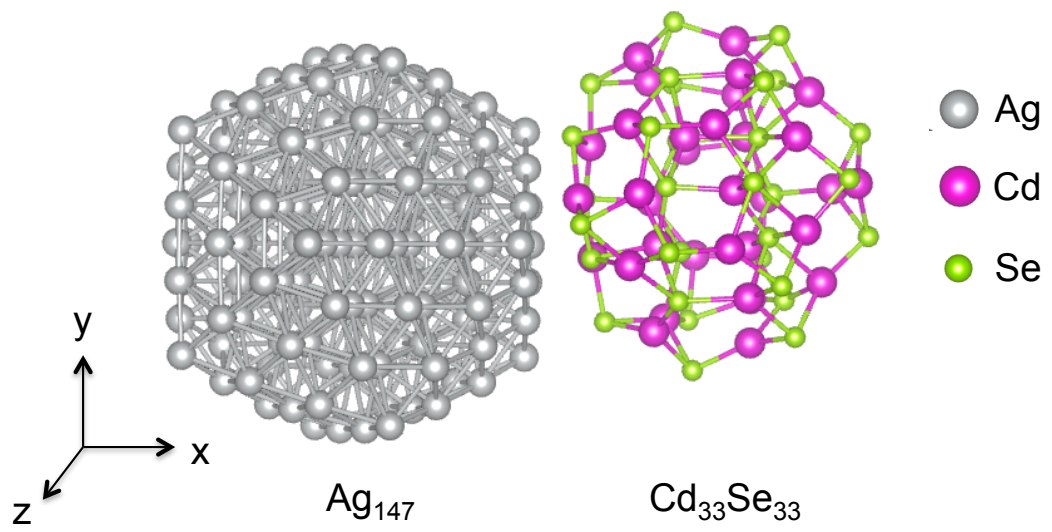

Figure 2: Structural model of the $\mathrm{Ag}_{147} \mathrm{Cd}_{33} \mathrm{Se}_{33}$ metal-semiconductor interface used in our calculations.

probabilities. DHET occurs from the occupied states of $\mathrm{Ag}_{147}$ to the unoccupied states of $\mathrm{Cd}_{33} \mathrm{Se}_{33}$ with a probability of $23.0 \%$, and DHHT occurs from the occupied states of $\mathrm{Cd}_{33} \mathrm{Se}_{33}$ to the unoccupied states of $\mathrm{Ag}_{147}$ with a probability of $21.4 \%$. Our results provide fundamental insights on the direct-transfer process, underscore the importance of both DHET and DHHT transitions, and have important implications for the field of plasmonics, as discussed below.

\section{Results and Discussion}

The relaxed combined $\mathrm{Ag}_{147}-\mathrm{Cd}_{33} \mathrm{Se}_{33}$ system is shown in Figure 2, with the interface normal along the $\mathrm{x}$-axis. The relaxation is carried out with DFT $^{39,40}$ using the GPAW code ${ }^{41,42}$ and structural optimizers in the ASE package. ${ }^{43}$ The wave functions are represented using a linear combination of atomic orbitals $(\mathrm{LCAO})^{44}$ and the projector augmented-wave (PAW) method $^{45}$ is utilized. Optical properties are computed using the LCAO-RT-TDDFT method as implemented in GPAW ${ }^{34,35}$ (see Methods section for more details). The photoabsorption spectrum is obtained using the $\delta$-kick technique ${ }^{46}$ to determine the position of the plasmon peak in our system.

Figure 3a shows the spectra for individual $\mathrm{Ag}_{147}$ and $\mathrm{Cd}_{33} \mathrm{Se}_{33}$ nanoparticles as well as the 
combined system when the electric field is applied along the $x$-axis, i.e. along the interface normal. Gaussian broadening of $0.2 \mathrm{eV}$ full width at half maximum was applied to the spectra. We observe a strong plasmon peak at $3.71 \mathrm{eV}$ for $\mathrm{Ag}_{147}$, which is consistent with previous calculations. ${ }^{34,35}$ On the other hand, we observe negligible absorption for $\mathrm{Cd}_{33} \mathrm{Se}_{33}$ in the energy range shown. Note that the used exchange-correlation functional is not expected to capture excitons in the semiconductor accurately. Therefore, we cannot rule out the possibility of photoabsorption due to exciton formation for $\mathrm{Cd}_{33} \mathrm{Se}_{33}$ in the energy range considered.

For the combined system, we observe a strong absorption peak at $3.60 \mathrm{eV}$. Compared to $\mathrm{Ag}_{147}$, this peak is red-shifted and broader, indicating dielectric screening due to the semiconductor and the presence of additional decay channels for the plasmon due to formation of the metal-semiconductor interface.

To study plasmon generation and decay to hot carriers at the $\mathrm{Ag}_{147}-\mathrm{Cd}_{33} \mathrm{Se}_{33}$ interface, we excite the combined system along the $x$-axis with a laser pulse at the plasmon frequency. The external electric field takes on the form $\mathcal{E}(t)=\mathcal{E}_{0} \sin \left(\omega_{p}\left(t-t_{0}\right)\right) \exp \left(-\sigma^{2}\left(t-t_{0}\right)^{2} / 2\right)$, where $t$ represents time, $\mathcal{E}_{0}$ is the field intensity (set to $1 \times 10^{-6}$ au to be weak enough to probe the linear-response regime), $\omega_{p}$ is the pulse frequency (set to the plasmon resonance frequency $3.60 \mathrm{eV}$ ), and $\sigma$ describes the pulse width (set to a value corresponding to pulse duration of $\sim 10 \mathrm{fs}$ ). The latter was chosen to cover the broad plasmon peak of the $\mathrm{Ag}_{147}-\mathrm{Cd}_{33} \mathrm{Se}_{33}$ system.

Figure $3 \mathrm{~b}$ shows the applied pulse and the dipole-moment response of the $\mathrm{Ag}_{147}-\mathrm{Cd}_{33} \mathrm{Se}_{33}$ system as a function of time. The pulse is turned on at $\sim 5 \mathrm{fs}$, reaches a maximum at $10 \mathrm{fs}$, and fades away after $15 \mathrm{fs}$. In response to this oscillating electric field, the dipole moment shows amplitude oscillations reaching a maximum at $\sim 11$ fs before decaying to a small value at $\sim 21$ fs (see Figure $\mathrm{S} 1$ in the Supporting Information for dipole moment over a longer time span). The dipole moment persists even after the pulse is switched off and the observed dipole-moment response is suggestive of a plasmon-mode excitation that decays to 
(a)

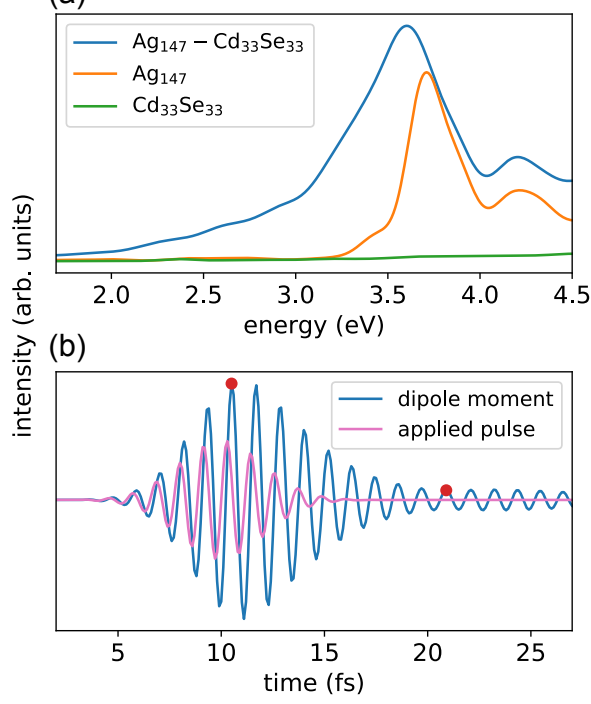

(c)
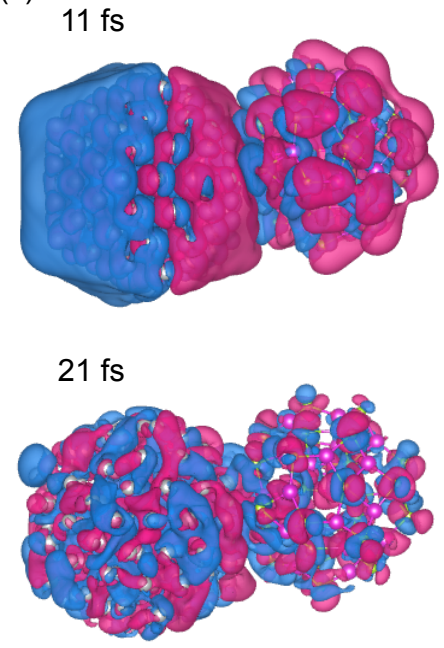

(d)
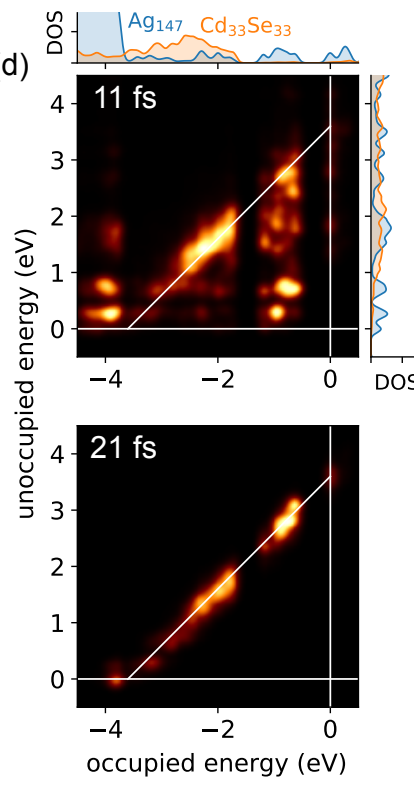

Figure 3: (a) Photoabsorption spectra of $\mathrm{Ag}_{147}-\mathrm{Cd}_{33} \mathrm{Se}_{33}, \mathrm{Ag}_{147}$, and $\mathrm{Cd}_{33} \mathrm{Se}_{33}$ systems. (b) Applied pulse and corresponding dipole-moment response in the time-domain for the $\mathrm{Ag}_{147^{-}}$ $\mathrm{Cd}_{33} \mathrm{Se}_{33}$ system. The two full red circles denote time points at 11 and $21 \mathrm{fs}$, corresponding to plasmon formation and decay to hot carriers, respectively. (c) Induced charge densities at 11 and 21 fs. Positive (purple) and negative (blue) isosurfaces are shown using the same isovalues in both the plots. (d) The corresponding Kohn-Sham electron-hole transition probability presented as transition-contribution maps at 11 and 21 fs (see text for more details). KohnSham energy eigenvalues are referenced to the Fermi level. The partial density of states (DOS) for $\mathrm{Ag}_{147}$ and $\mathrm{Cd}_{33} \mathrm{Se}_{33}$ are also shown.

single-particle excitations (hot carriers). ${ }^{19}$

To assess the plasmonic character, we visualize the induced charge density in real space at 11 and $21 \mathrm{fs}$ (Figure 3c). At $11 \mathrm{fs}$ the charge density undergoes collective oscillations, reminiscent of the classical picture of a plasmon in which charge moves from one side of the metal nanoparticle surface to the other. We also observe a significant portion of the charge density on the $\mathrm{Cd}_{33} \mathrm{Se}_{33}$ cluster, indicating that the excitation can delocalize onto the chemically attached acceptor. On the other hand, at $21 \mathrm{fs}$, the charge-density distribution is mostly fragmented showing no clear feature of collective oscillations, indicative of plasmon decay and consequent generation of hot carriers.

We analyzed plasmon formation and hot-carrier generation in terms of the Kohn-Sham (KS) electron-hole transition contributions at 11 and $21 \mathrm{fs}$, respectively. To this end, we 
construct the KS transition density matrix, $\delta \rho_{i a}^{x}(t)=\rho_{i a}^{x}(t)-\rho_{i a}^{x}(0)$, in the basis of occupied (i) and unoccupied (a) KS states as described in Ref. 35. Then, following Ref. 47, we consider the transition probability $P_{i a}(t)=\left|\delta \rho_{i a}^{x}(t) / \sqrt{f_{i}-f_{a}}\right|^{2}$, where $f_{i}$ and $f_{a}$ are the ground-state occupations of KS states $i$ and $a$, respectively. We present this quantity as a transition-contribution map (TCM), which is a useful visualization for large systems where resonances arise from the superposition of multiple electron-hole excitations. ${ }^{35,48,49}$ Briefly, in the TCM, the elements of the matrix $P_{i a}(t)$ are plotted as Gaussian-broadened dots at the corresponding KS eigenvalue pair $\left(\epsilon_{i}, \epsilon_{a}\right)$ on a two-dimensional plane spanned by the energy axes for occupied and unoccupied states, see Ref. 35 for details on TCM construction.

TCMs of the $\mathrm{Ag}_{147}-\mathrm{Cd}_{33} \mathrm{Se}_{33}$ system at 11 and 21 fs are shown in Figure 3d, along with the partial density of states (DOS) for $\mathrm{Ag}_{147}$ and $\mathrm{Cd}_{33} \mathrm{Se}_{33}$ components (see Figure $\mathrm{S} 2$ in the Supporting Information for a larger DOS plot). In the TCM at $11 \mathrm{fs,} \mathrm{we} \mathrm{notice} \mathrm{a}$ strong contribution arising from low-energy transitions, whose eigenvalue differences are much smaller than the frequencies included in the laser pulse. This is indicated by the many features that appear in the TCM in the bottom right of the plot. Such a constructive coupling of low-energy transitions is a signature of the localized surface plasmon resonance. ${ }^{35}$ Thus, the TCM at $11 \mathrm{fs}$ mainly reflects the formation of a plasmon in the $\mathrm{Ag}_{147}-\mathrm{Cd}_{33} \mathrm{Se}_{33}$ system, consistent with the strong dipole-moment oscillations and the cumulative charge density computed at $11 \mathrm{fs} .{ }^{47}$

On the other hand, the TCM at $21 \mathrm{fs}$ shows a different behavior. We observe a single prominent contribution stemming from transitions whose eigenvalue differences are equal to the frequencies of the laser pulse. These resemble single-particle excitations and are consistent with the hot-carrier picture emerging in recent literature. ${ }^{19,47}$ The absence of constructive low-energy features that constitute a plasmon suggests that the plasmon formed at $11 \mathrm{fs}$ has decayed to yield hot carriers, ${ }^{47}$ consistent with the weak dipole moment and the fragmented charge density computed at $21 \mathrm{fs}$.

From the transition probability $P_{i a}(t)$, we can compute the hot-carrier distributions as 
well. Following Ref. 47, the induced occupations of electrons in state $a$ and holes in state $i$ are given by $P_{a}^{\mathrm{e}}(t)=\sum_{i} P_{i a}(t)$ and $P_{i}^{\mathrm{h}}(t)=\sum_{a} P_{i a}(t)$, respectively. The corresponding hot-electron distribution $P_{\mathrm{e}}(E, t)$ presents the occupation with respect to the eigenvalues of the states, $P_{\mathrm{e}}(E, t)=\sum_{a} P_{a}^{\mathrm{e}}(t) G_{a}(E)$, where a Gaussian broadening function is employed. The hot-hole distribution $P_{\mathrm{h}}(E, t)$ is computed analogously. The total number of generated carriers is given by $N(t)=\sum_{a} P_{a}^{\mathrm{e}}(t)=\sum_{i} P_{i}^{\mathrm{h}}(t)=\sum_{i a} P_{i a}(t)$, which is equal for electrons and holes.

Figure 4a shows the total hot-carrier distributions, $P_{\mathrm{e}}(E, t)$ and $P_{\mathrm{h}}(E, t)$, in the $\mathrm{Ag}_{147^{-}}$ $\mathrm{Cd}_{33} \mathrm{Se}_{33}$ system at $21 \mathrm{fs}$, i.e., immediately after plasmon decay. The distribution reveals that hot holes and electrons with energies up to the laser pulse frequency $(\sim 4 \mathrm{eV})$ are generated. For reference, we consider the joint density of states and show also the electron and hole distributions of the transitions that are covered by the used pulse in the joint DOS. In comparison to the hot-carrier distributions, the joint-DOS-based distributions have different relative weights demonstrating that the actual matrix elements play an important role in the distributions. The hole and electron distributions exhibit slightly different shapes due to the spectral width of the pulse.

The total hot-carrier distribution can be divided into four parts resulting from four partial processes: (i) $P_{\mathrm{e} / \mathrm{h}}^{\mathrm{Ag} \rightarrow \mathrm{Ag}}$ transitions from occupied states of $\mathrm{Ag}$ to unoccupied states of $\mathrm{Ag}$, (ii) $P_{\mathrm{e} / \mathrm{h}}^{\mathrm{CdSe} \rightarrow \mathrm{CdSe}}$ transitions from occupied states of CdSe to unoccupied states of CdSe, (iii) $P_{\mathrm{e} / \mathrm{h}}^{\mathrm{Ag} \rightarrow \mathrm{CdSe}}$ transitions from occupied states of $\mathrm{Ag}$ to unoccupied states of CdSe, and (iv) $P_{\mathrm{e} / \mathrm{h}}^{\mathrm{CdSe} \rightarrow \mathrm{Ag}}$ transitions from occupied states of CdSe to unoccupied states of $\mathrm{Ag}$. Therefore, $P_{\mathrm{e} / \mathrm{h}}=P_{\mathrm{e} / \mathrm{h}}^{\mathrm{Ag} \rightarrow \mathrm{Ag}}+P_{\mathrm{e} / \mathrm{h}}^{\mathrm{CdSe} \rightarrow \mathrm{CdSe}}+P_{\mathrm{e} / \mathrm{h}}^{\mathrm{Ag} \rightarrow \mathrm{CdSe}}+P_{\mathrm{e} / \mathrm{h}}^{\mathrm{CdSe} \rightarrow \mathrm{Ag}}$.

Here, the separation into $\mathrm{Ag}$ and CdSe subsystems is done by dividing the simulation cell into two complementary spatial regions determined by the boundary between Ag and CdSe atoms. For each $\mathrm{KS}$ state $n, \mathrm{Ag}$ and CdSe weights are calculated from the groundstate KS wave function $\psi_{n}(\mathbf{r})$ as $w_{n}^{\mathrm{Ag}}=\int_{\mathbf{r} \in \mathrm{Ag}}\left|\psi_{n}(\mathbf{r})\right|^{2} \mathrm{~d} \mathbf{r}$, and $w_{n}^{\mathrm{CdSe}}=\int_{\mathbf{r} \in \mathrm{CdSe}}\left|\psi_{n}(\mathbf{r})\right|^{2} \mathrm{~d} \mathbf{r}$, respectively, satisfying $w_{n}^{\mathrm{Ag}}+w_{n}^{\mathrm{CdSe}}=1$. Then, the partial hot-carrier distributions are 

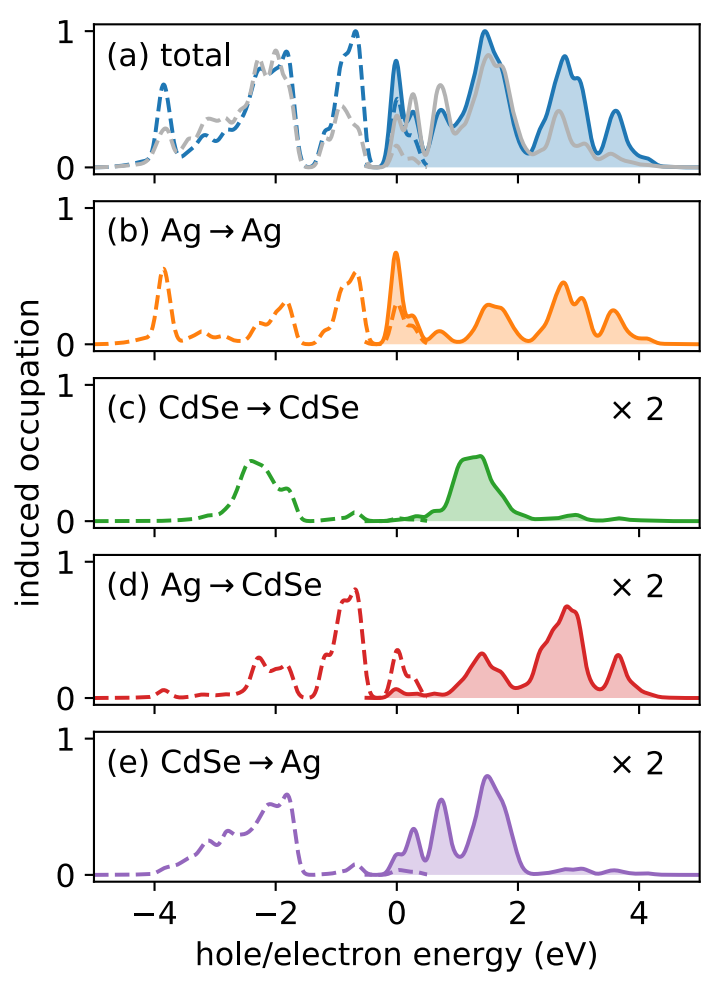

Figure 4: (a) Total (in blue) and joint-DOS-based (in grey), and (b-e) partial hot-electron (solid lines and shaded) and hot-hole (dotted lines and unshaded) distributions for the $\mathrm{Ag}_{147^{-}}$ $\mathrm{Cd}_{33} \mathrm{Se}_{33}$ system. The total probabilities of the four partial transitions are $43.4 \%, 12.2 \%$, $23.0 \%$ and $21.4 \%$, from b to e, respectively. The relative probabilities in (c-e) are multiplied by a factor 2 for visual clarity, as indicated.

obtained by using the weights for occupied $(i)$ and unoccupied $(a)$ states in the transition probability. As an example, the hot-electron distribution $P_{\mathrm{e}}^{\mathrm{Ag} \rightarrow \mathrm{CdSe}}$ is obtained from the weighted transition probability $P_{i a}^{\mathrm{Ag} \rightarrow \mathrm{CdSe}}(t)=w_{i}^{\mathrm{Ag}} w_{a}^{\mathrm{CdSe}} P_{i a}(t)$ analogously as above, $P_{\mathrm{e}}^{\mathrm{Ag} \rightarrow \mathrm{CdSe}}(E, t)=\sum_{i a} P_{i a}^{\mathrm{Ag} \rightarrow \mathrm{CdSe}}(t) G_{a}(E)$. The hot-hole distribution, $P_{\mathrm{h}}^{\mathrm{Ag} \rightarrow \mathrm{CdSe}}$ is computed analogously. Using similar arguments, we obtain $P_{\mathrm{e} / \mathrm{h}}^{\mathrm{Ag} \rightarrow \mathrm{Ag}}, P_{\mathrm{e} / \mathrm{h}}^{\mathrm{CdSe} \rightarrow \mathrm{CdSe}}$, and $P_{\mathrm{e} / \mathrm{h}}^{\mathrm{CdSe} \rightarrow \mathrm{Ag}}$. The number of carriers generated by a partial process is calculated in equivalent fashion, for example $N^{\mathrm{Ag} \rightarrow \mathrm{CdSe}}(t)=\sum_{i a} P_{i a}^{\mathrm{Ag} \rightarrow \mathrm{CdSe}}(t)$. Note that the present spatial division approach can be developed further to account also for the coherent contributions from the off-diagonal of the electron-electron part of the time-dependent density matrix.

The total probability of a particular partial process is calculated as the ratio between the number of carriers generated by the partial process and the total number of carriers generated 
by all processes. This probability value is given as a percentage in the discussion below and should not be confused with the previously-mentioned transition probability between two particular KS states.

Figure $4 \mathrm{~b}$ shows the $P_{\mathrm{e} / \mathrm{h}}^{\mathrm{Ag} \rightarrow \mathrm{Ag}}$ partial hot-carrier distribution. We observe that a large part (corresponding to a total probability of $43.4 \%$ ) of the plasmon decays within the $\mathrm{Ag}_{147}$ nanoparticle, inducing intraband $(s p \rightarrow s p)$ and interband $(d \rightarrow s p)$ transitions commonly observed in Ag nanoparticles. ${ }^{19,35,47}$ We do not, however, consider subsequent transport processes involving hot carriers generated in this manner that could facilitate their transfer to the semiconductor; this would constitute the indirect-transfer process. These transport processes are expected to occur on timescales beyond those relevant to the present work. ${ }^{13}$

Figure $4 \mathrm{c}$ shows the $P_{\mathrm{e} / \mathrm{h}}^{\mathrm{CdSe} \rightarrow \mathrm{CdSe}}$ partial hot-carrier distribution. This transition, commonly known as intra-acceptor excitation (see Figure 1c), occurs when plasmons directly excite electrons from occupied states of the acceptor to unoccupied states of the acceptor. ${ }^{50,51}$ Our computation shows that about $12.2 \%$ of the plasmon decays through this channel in our structure.

We also examine the direct-transfer process in our simulations. Figures 4 d,e show the $P_{\mathrm{e} / \mathrm{h}}^{\mathrm{Ag} \rightarrow \mathrm{CdSe}}$ and $P_{\mathrm{e} / \mathrm{h}}^{\mathrm{CdSe} \rightarrow \mathrm{Ag}}$ partial hot-carrier distributions, corresponding to the direct-transfer processes, DHET and DHHT, respectively. They both lead to different carrier distributions in the system. We find that the probabilities of these two processes are $23.0 \%$ and $21.4 \%$, respectively. Thus, direct transfer contributes up to $44.4 \%$ of the total plasmon decay, demonstrating that it could play a significant role at metal-acceptor interfaces.

To check if the choice of the plasmon decay time point has any influence on the results, we computed the probabilities of the four partial processes as a function of time (see Figure S3 in the Supporting Information). Our results showed little variation in the probability values beyond $\sim 21 \mathrm{fs}$, indicating that the plasmon decay has reached a steady state beyond this time point.

We carried out additional simulations with another pulse with a longer duration of $\sim 24 \mathrm{fs}$ 
tuned to the plasmon frequency. For this case, the total probabilities of the four partial transitions, $P_{\mathrm{e} / \mathrm{h}}^{\mathrm{Ag} \rightarrow \mathrm{Ag}}, P_{\mathrm{e} / \mathrm{h}}^{\mathrm{CdSe} \rightarrow \mathrm{CdSe}}, P_{\mathrm{e} / \mathrm{h}}^{\mathrm{Ag} \rightarrow \mathrm{CdSe}}$ and $P_{\mathrm{e} / \mathrm{h}}^{\mathrm{CdS} \rightarrow \mathrm{Ag}}$, computed after plasmon decay are $41.2 \%, 12.6 \%, 24.5 \%$, and $21.7 \%$, respectively. These values do not differ significantly from the ones obtained for the 10-fs pulse.

We note that the values obtained here are for a particular interface configuration. In experiments, one can, however, expect several different interface structures to be present (see Figure S4 in the Supporting Information for additional simulations with different system sizes). Furthermore, different material combinations can be employed in experiments. In all of these cases, one could obtain the corresponding probabilities by utilizing the method described here with the relevant structure as input. This then becomes case-specific and goes beyond the scope of this study. Although the probabilities are expected to change, the underlying physics of plasmon decay and hot-carrier generation can be expected to remain the same.

We expect our results to have important implications for the field of plasmonics. An approximate way to obtain the contribution of the direct-transfer process in experiments is by measuring the plasmon linewidth in the photoabsorption spectra with and without the acceptor attached, and by taking their difference (see Refs. 30 and 52). This method cannot, however, distinguish between the two direct-transfer processes, DHET and DHHT. As such, the relative importance of DHET and DHHT has remained unclear. Consequently, in the existing literature, the direct-transfer process is commonly discussed in terms of DHET, which produces hot electrons in the acceptor, while DHHT, which produces hot holes in the acceptor, is often overlooked. Here, we find that both of these transitions occur with similar probability. This could also be the case in other systems of interest. Therefore, it would be necessary to treat both of these possibilities and consider their probabilities in future studies.

It is also expected that an increase in the direct-transfer contribution will improve the efficiency of a plasmonic hot-carrier device. While this statement could be true for applications such as photocatalysis and photon upconversion, it may not necessarily hold true for 

carrier separation is important. Our results show that although a significant proportion of carriers are generated through the direct-transfer process at the metal-semiconductor interface ( $44.4 \%$ of total), the difference in the probabilities of DHET and DHHT is rather small ( $23.0 \%$ vs. $21.4 \%$ of total), resulting in low net-carrier separation, i.e. a large number of electrons generated in the acceptor by DHET is counterbalanced by a large number of holes generated in the acceptor by DHHT. Therefore, for direct transfer to have a positive impact in such applications, one of these transitions must be favored at the expense of the other.

Given the importance of DHET and DHHT toward efficient charge transfer across metalacceptor interfaces, the development of strategies for controlling and tuning these two partial processes is critical for obtaining useful plasmonic hot-carrier devices. Specifically, we considered via the presented approach whether such tuning is possible by altering the interface electronic structure with strain. We applied compressive strains of $-6 \%$ and $-3 \%$ on the $\mathrm{Ag}_{147}$ nanoparticle, while maintaining the $\mathrm{Cd}_{33} \mathrm{Se}_{33}$ cluster unstrained. The resulting $\mathrm{Ag}_{14 \tau^{-}}$ $\mathrm{Cd}_{33} \mathrm{Se}_{33}$ configurations are relaxed and the optical properties are studied as described in the Methods section.

The plasmon peak positions shifted only slightly from $3.60 \mathrm{eV}$ for the unstrained system to $3.59 \mathrm{eV}$ and $3.63 \mathrm{eV}$ for the $-3 \%$ and $-6 \%$ strained cases, respectively (Figure $5 \mathrm{a}$ ). The plasmon peak values were changed accordingly in our laser simulations, while all the other parameters were kept the same as described earlier. Interestingly, with compression, we find that there is a crossover between the number of carriers generated via DHET and DHHT (Figure 5b). We also find that the absolute difference in the number of carriers induced by DHET and DHHT increases from $8.60 \times 10^{-5}$ to about $1.80 \times 10^{-4}$ (by a factor of $\sim 2$ ). This result shows that the application of external stimuli, such as strain, could be promising for controlling DHET and DHHT, and thus for obtaining a handle on the direct-transfer process.

While we have attempted to analyze the nature of the above observed changes, we find 


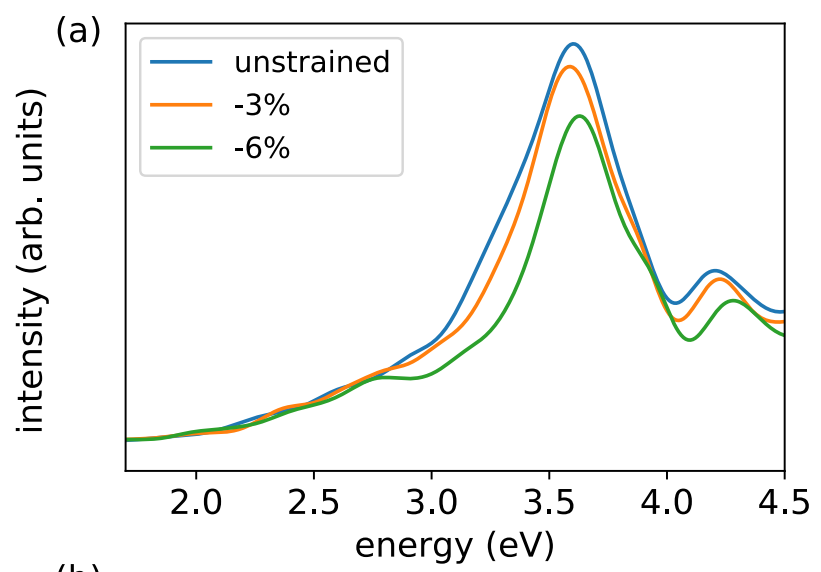

(b)

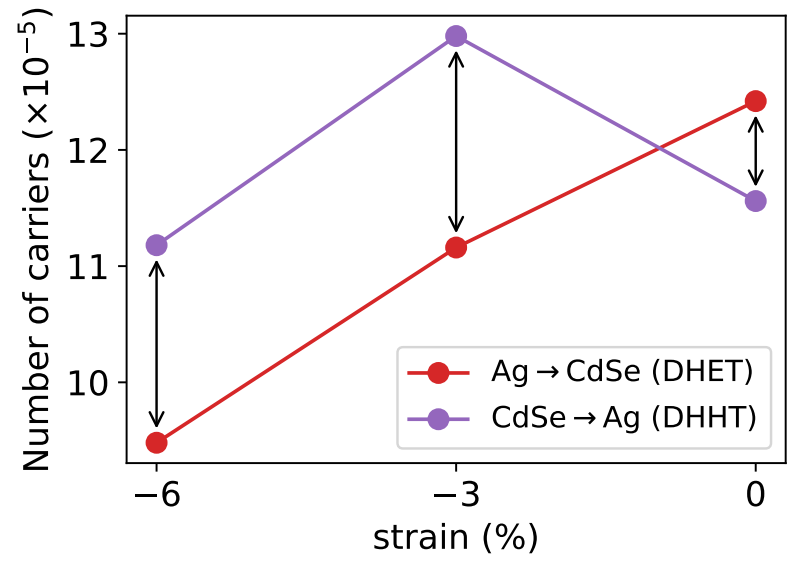

Figure 5: (a) Photoabsorption spectra of the unstrained and strained $\mathrm{Ag}_{147} \mathrm{Cd}_{33} \mathrm{Se}_{33}$ systems. (b) Tuning the number of carriers induced by the two direct-transfer transitions, DHET (Ag $\rightarrow$ CdSe) and DHHT (CdSe $\rightarrow \mathrm{Ag}$ ), by applying compressive strain on the $\mathrm{Ag}_{147}$ nanoparticle. The corresponding differences between them are indicated by double-headed arrows.

that a complicated interplay of the changes in the matrix elements and the density of states could be the cause. It should be noted that strain also lead to changes in phonon interactions, which may impact the processes considered here. Although neglected here, care must be taken to account for such effects in the future. Other experimentally controllable parameters such as the size, shape, and composition of the metallic nanoparticle could also provide enhanced control. For example, the two processes might be controllable independently, and the difference in the probabilities of DHET and DHHT could be further improved. These topics are planned to be examined in future work. 


\section{Conclusions}

In summary, we report a TDDFT study of the direct charge-transfer process at a model $\mathrm{Ag}_{147} \mathrm{Cd}_{33} \mathrm{Se}_{33}$ metal-semiconductor interface. Our work isolates and quantifies this fundamental charge-transfer mechanism at metal-acceptor interfaces that has been difficult to access through experiments. For a 10-femtosecond Gaussian laser pulse tuned to the plasmon frequency, our TDDFT simulations clearly reveal plasmon formation in the $\mathrm{Ag}_{147}-\mathrm{Cd}_{33} \mathrm{Se}_{33}$ system, which decays within $10 \mathrm{fs}$ and induces direct transfer with a probability of $44.4 \%$. The calculations show that the direct-transfer step to consist of two key processes that inject electrons and holes into the acceptor, namely the direct hot-electron transfer (DHET) and the direct hot-hole transfer (DHHT). These occur with considerable probability of $23.0 \%$ and $21.4 \%$, respectively. Our analysis further demonstrate that the probabilities of DHET and DHHT are sensitive to external stimuli such as strain. The methodology described here could not only help understand and determine the probability of the direct-transfer process at a given metal-acceptor interface, but also assist in designing interfaces that can favor the direct-transfer pathway over the indirect-transfer pathway that is generally lossy. Collectively, our work has important implications for the field of plasmonics and could lead to more efficient plasmonic hot-carrier devices in the future.

\section{Methods}

Relaxation calculations. First, the $\mathrm{Ag}_{147}$ nanoparticle and the $\mathrm{Cd}_{33} \mathrm{Se}_{33}$ cluster were relaxed separately. Then, the combined $\mathrm{Ag}_{147^{-}} \mathrm{Cd}_{33} \mathrm{Se}_{33}$ system was assembled and relaxed keeping the atoms of the $\mathrm{Ag}_{147}$ nanoparticle fixed, while the atoms of the $\mathrm{Cd}_{33} \mathrm{Se}_{33}$ cluster were allowed to relax. All the structures were relaxed to less than $0.03 \mathrm{eV} / \AA$ residual forces. The Perdew-Burke-Ernzerhof (PBE) exchange-correlation (xc) functional was used. ${ }^{53}$ A grid spacing parameter of $0.3 \AA$ for densities and potentials was used, and the structure was surrounded by a vacuum spacing of at least $6 \AA$. The default double- $\zeta$ polarized basis sets 
were used. Within the PAW description, 11, 12, and 6 valence electrons were included to model Ag, Cd, and Se atoms, respectively.

TDDFT calculations. The orbital-dependent GLLB-SC xc potential ${ }^{54}$ was used to improve the description of $d$-electron states in silver. ${ }^{34,55}$ A Fermi-Dirac smearing of $0.15 \mathrm{eV}$ was used to facilitate the convergence with this xc potential. The basis set of Ag atoms contained the diffuse $5 p$ orbital and its split-valence function instead of the standard $p$-type

polarization function in order to describe the plasmon accurately in the Ag nanoparticle. ${ }^{34,56}$ A time step of 20 as was used in the time propagation. Other numerical parameters were similar to those in the relaxation calculations. More details on the LCAO-RT-TDDFT methodology can be found in Ref. 35.

\section{Supporting Information Available}

The Supporting Information is available free of charge on the ACS Publications website at DOI:Xxx

Dipole moment plot at longer timescales; partial density of states for the $\mathrm{Ag}_{147}$ and $\mathrm{Cd}_{33} \mathrm{Se}_{33}$ components; number of carriers generated and probabilities of the four partial processes as a function of time; probabilities of the four partial processes obtained with different systems; structure file in $x y z$-format

\section{Corresponding author}

David J. Norris, E-mail: dnorris@ethz.ch

\section{ORCID}

Priyank V. Kumar: 0000-0002-8203-7223

Tuomas P. Rossi: 0000-0002-8713-4559 
Mikael Kuisma: 0000-0001-8323-3405

Paul Erhart: 0000-0002-2516-6061

Martti Puska: 0000-0002-8419-3289

David J. Norris: 0000-0002-3765-0678

\section{Acknowledgement}

P.V.K. thanks the Marie Curie ETH Zurich Postdoctoral Fellowship for financial support. T.P.R. and P.E. acknowledge support from the Knut and Alice Wallenberg Foundation and the Swedish Research Council. P.V.K. and T.P.R. also thank the network in Condensed Matter and Materials Physics (Aalto University) for support. M.K. acknowledges funding from Academy of Finland under grant Nr. 295602. M.P. and T.P.R. thank the Academy of Finland for support through its Centres of Excellence Programme under Project No. 251748. P.V.K. thanks Dr. A.K. Michel for fruitful discussions. Computational resources were provided by the ETH High-Performance Computing Cluster (Euler), and by the Swedish National Infrastructure for Computing at PDC (Stockholm), NSC (Linköping), and C3SE (Gothenburg).

\section{References}

1. Brongersma, M. L.; Halas, N. J.; Nordlander, P. Plasmon-Induced Hot Carrier Science and Technology. Nat. Nanotechnol. 2015, 10, 25-34.

2. Linic, S.; Aslam, U.; Boerigter, C.; Morabito, M. Photochemical Transformations on Plasmonic Metal Nanoparticles. Nat. Mater. 2015, 14, 567-576.

3. Atwater, H. A.; Polman, A. Plasmonics for Improved Photovoltaic Devices. Nat. Mater. 2010, 9, 205-213.

4. Knight, M. W.; Sobhani, H.; Nordlander, P.; Halas, N. J. Photodetection with Active Optical Antennas. Science 2011, 332, 702-704. 
5. Chalabi, H.; Schoen, D.; Brongersma, M. L. Hot-Electron Photodetection with a Plasmonic Nanostripe Antenna. Nano Lett. 2014, 14, 1374-1380.

6. Naik, G. V.; Welch, A. J.; Briggs, J. A.; Solomon, M. L.; Dionne, J. A. Hot-CarrierMediated Photon Upconversion in Metal-Decorated Quantum Wells. Nano Lett. 2017, $17,4583-4587$.

7. Mubeen, S.; Lee, J.; Singh, N.; Krämer, S.; Stucky, G. D.; Moskovits, M. An Autonomous Photosynthetic Device in Which All Charge Carriers Derive from Surface Plasmons. Nat. Nanotechnol. 2013, 8, 247-251.

8. Kale, M. J.; Avanesian, T.; Christopher, P. Direct Photocatalysis by Plasmonic Nanostructures. ACS Catal. 2014, 4, 116-128.

9. Narang, P.; Sundararaman, R.; Atwater, H. A. Plasmonic Hot Carrier Dynamics in SolidState and Chemical Systems for Energy Conversion. Nanophotonics 2016, 5, 96-111.

10. Christopher, P.; Moskovits, M. Hot Charge Carrier Transmission from Plasmonic Nanostructures. Annu. Rev. Phys. Chem. 2017, 68, 379-398.

11. Zheng, B. Y.; Zhao, H.; Manjavacas, A.; McClain, M.; Nordlander, P.; Halas, N. J. Distinguishing between Plasmon-induced and Photoexcited Carriers in a Device Geometry. Nat. Commun. 2015, 6, 7797.

12. Cortés, E.; Xie, W.; Cambiasso, J.; Jermyn, A. S.; Sundararaman, R.; Narang, P.; Schlücker, S.; Maier, S. A. Plasmonic Hot Electron Transport Drives Nano-Localized Chemistry. Nat. Commun. 2017, 8, 14880.

13. Ratchford, D. C.; Dunkelberger, A. D.; Vurgaftman, I.; Owrutsky, J. C.; Pehrsson, P. E. Quantification of Efficient Plasmonic Hot-Electron Injection in Gold Nanoparticle- $\mathrm{TiO}_{2}$ Films. Nano Lett. 2017, 17, 6047-6055. 
14. Tan, S.; Liu, L.; Dai, Y.; Ren, J.; Zhao, J.; Petek, H. Ultrafast Plasmon-Enhanced Hot Electron Generation at Ag Nanocluster/Graphite Heterojunctions. J. Am. Chem. Soc. 2017, 139, 6160-6168.

15. White, T. P.; Catchpole, K. R. Plasmon-Enhanced Internal Photoemission for Photovoltaics: Theoretical Efficiency Limits. Appl. Phys. Lett. 2012, 101, 073905.

16. Sundararaman, R.; Narang, P.; Jermyn, A. S.; Goddard, W. A.; Atwater, H. A. Theoretical Predictions for Hot-Carrier Generation from Surface Plasmon Decay. Nat. Commun. 2014, 5, 5788 .

17. Manjavacas, A.; Liu, J. G.; Kulkarni, V.; Nordlander, P. Plasmon-Induced Hot Carriers in Metallic Nanoparticles. ACS Nano 2014, 8, 7630-7638.

18. Bernardi, M.; Mustafa, J.; Neaton, J. B.; Louie, S. G. Theory and Computation of Hot Carriers Generated by Surface Plasmon Polaritons in Noble Metals. Nat. Commun. 2015, 6, 7044 .

19. Ma, J.; Wang, Z.; Wang, L.-W. Interplay Between Plasmon and Single-Particle Excitations in a Metal Nanocluster. Nat. Commun. 2015, 6, 10107.

20. Brown, A. M.; Sundararaman, R.; Narang, P.; Goddard, W. A.; Atwater, H. A. Nonradiative Plasmon Decay and Hot Carrier Dynamics: Effects of Phonons, Surfaces, and Geometry. ACS Nano 2016, 10, 957-966.

21. Brown, A. M.; Sundararaman, R.; Narang, P.; Schwartzberg, A. M.; Goddard, W. A.; Atwater, H. A. Experimental and Ab Initio Ultrafast Carrier Dynamics in Plasmonic Nanoparticles. Phys. Rev. Lett. 2017, 118, 087401.

22. Besteiro, L. V.; Kong, X.-T.; Wang, Z.; Hartland, G.; Govorov, A. O. Understanding Hot-Electron Generation and Plasmon Relaxation in Metal Nanocrystals: Quantum and Classical Mechanisms. ACS Photon. 2017, 4, 2759-2781. 
23. Yan, L.; Wang, F.; Meng, S. Quantum Mode Selectivity of Plasmon-Induced Water Splitting on Gold Nanoparticles. ACS Nano 2016, 10, 5452-5458.

24. Wu, K.; Chen, J.; McBride, J. R.; Lian, T. Efficient Hot-Electron Transfer by a PlasmonInduced Interfacial Charge-Transfer Transition. Science 2015, 349, 632-635.

25. Tan, S.; Argondizzo, A.; Ren, J.; Liu, L.; Zhao, J.; Petek, H. Plasmonic Coupling at a Metal/Semiconductor Interface. Nat. Photon. 2017, 11, 806-812.

26. Tan, S.; Dai, Y.; Zhang, S.; Liu, L.; Zhao, J.; Petek, H. Coherent Electron Transfer at the $\mathrm{Ag} /$ Graphite Heterojunction Interface. Phys. Rev. Lett. 2018, 120, 126801.

27. Li, Y.; DiStefano, J. G.; Murthy, A. A.; Cain, J. D.; Hanson, E. D.; Li, Q.; Castro, F. C.; Chen, X.; Dravid, V. P. Superior Plasmonic Photodetectors Based on Au@MoS ${ }_{2}$ CoreShell Heterostructures. ACS Nano 2017, 11, 10321-10329.

28. Boerigter, C.; Campana, R.; Morabito, M.; Linic, S. Evidence and Implications of Direct Charge Excitation as the Dominant Mechanism in Plasmon-Mediated Photocatalysis. Nat. Commun. 2016, 7, 10545.

29. Boerigter, C.; Aslam, U.; Linic, S. Mechanism of Charge Transfer from Plasmonic Nanostructures to Chemically Attached Materials. ACS Nano 2016, 10, 6108-6115.

30. Foerster, B.; Joplin, A.; Kaefer, K.; Celiksoy, S.; Link, S.; Sönnichsen, C. Chemical Interface Damping Depends on Electrons Reaching the Surface. ACS Nano 2017, 11, 2886-2893.

31. Kale, M. J.; Christopher, P. Plasmons at the Interface. Science 2015, 349, 587-588.

32. Douglas-Gallardo, O. A.; Berdakin, M.; Sánchez, C. G. Atomistic Insights into Chemical Interface Damping of Surface Plasmon Excitations in Silver Nanoclusters. J. Phys. Chem. C 2016, 120, 24389-24399. 
33. Runge, E.; Gross, E. K. U. Density-Functional Theory for Time-Dependent Systems. Phys. Rev. Lett. 1984, 52, 997-1000.

34. Kuisma, M.; Sakko, A.; Rossi, T. P.; Larsen, A. H.; Enkovaara, J.; Lehtovaara, L.; Rantala, T. T. Localized Surface Plasmon Resonance in Silver Nanoparticles: Atomistic First-Principles Time-Dependent Density-Functional Theory Calculations. Phys. Rev. B 2015, 91, 115431.

35. Rossi, T. P.; Kuisma, M.; Puska, M. J.; Nieminen, R. M.; Erhart, P. Kohn-Sham Decomposition in Real-Time Time-Dependent Density-Functional Theory: An Efficient Tool for Analyzing Plasmonic Excitations. J. Chem. Theory Comput. 2017, 13, 4779-4790.

36. Puzder, A.; Williamson, A. J.; Gygi, F.; Galli, G. Self-Healing of CdSe Nanocrystals: First-Principles Calculations. Phys. Rev. Lett. 2004, 92, 217401.

37. Kilina, S.; Ivanov, S.; Tretiak, S. Effect of Surface Ligands on Optical and Electronic Spectra of Semiconductor Nanoclusters. J. Am. Chem. Soc. 2009, 131, 7717-7726.

38. Zhou, M.; Li, J.; Ye, Z.; Ma, C.; Wang, H.; Huo, P.; Shi, W.; Yan, Y. Transfer Charge and Energy of Ag@CdSe QDs-rGO Core-Shell Plasmonic Photocatalyst for Enhanced Visible Light Photocatalytic Activity. ACS Appl. Mater. Interfaces 2015, 7, 28231-28243.

39. Hohenberg, P.; Kohn, W. Inhomogeneous Electron Gas. Phys. Rev. 1964, 136, B864B871.

40. Kohn, W.; Sham, L. J. Self-Consistent Equations Including Exchange and Correlation Effects. Phys. Rev. 1965, 140, A1133-A1138.

41. Mortensen, J. J.; Hansen, L. B.; Jacobsen, K. W. Real-Space Grid Implementation of the Projector Augmented Wave Method. Phys. Rev. B 2005, 71, 035109.

42. Enkovaara, J.; Rostgaard, C.; Mortensen, J. J.; Chen, J.; Dułak, M.; Ferrighi, L.; Gavnholt, J.; Glinsvad, C.; Haikola, V.; Hansen, H. A.; Kristoffersen, H. H.; Kuisma, M.; 
Larsen, A. H.; Lehtovaara, L.; Ljungberg, M.; Lopez-Acevedo, O.; Moses, P. G.; Ojanen, J.; Olsen, T.; Petzold, V. et al. Electronic Structure Calculations with GPAW: A Real-Space Implementation of the Projector Augmented-Wave Method. J. Phys. Condens. Matter 2010, 22, 253202.

43. Larsen, A.; Mortensen, J.; Blomqvist, J.; Castelli, I.; Christensen, R.; Dulak, M.; Friis, J.; Groves, M.; Hammer, B.; Hargus, C.; Hermes, E.; Jennings, P.; Jensen, P.; Kermode, J.; Kitchin, J.; Kolsbjerg, E.; Kubal, J.; Kaasbjerg, K.; Lysgaard, S.; Maronsson, J. et al. The Atomic Simulation Environment - A Python Library For Working with Atoms. J. Phys. Condens. Matter 2017, 29, 273002.

44. Larsen, A. H.; Vanin, M.; Mortensen, J. J.; Thygesen, K. S.; Jacobsen, K. W. Localized Atomic Basis Set in the Projector Augmented Wave Method. Phys. Rev. B 2009, 80, 195112.

45. Blöchl, P. E. Projector Augmented-Wave Method. Phys. Rev. B 1994, 50, 17953-17979.

46. Yabana, K.; Bertsch, G. F. Time-Dependent Local-Density Approximation in Real Time. Phys. Rev. B 1996, 54, 4484-4487.

47. Rossi, T. P.; Erhart, P.; Kuisma, M. Hot-Carrier Generation in Plasmonic Nanoparticles. unpublished work

48. Baseggio, O.; De Vetta, M.; Fronzoni, G.; Stener, M.; Sementa, L.; Fortunelli, A.; Calzolari, A. Photoabsorption of Icosahedral Noble Metal Clusters: An Efficient TDDFT Approach to Large-Scale Systems. J. Phys. Chem. C 2016, 120, 12773-12782.

49. Malola, S.; Lehtovaara, L.; Enkovaara, J.; Häkkinen, H. Birth of the Localized Surface Plasmon Resonance in Monolayer-Protected Gold Nanoclusters. ACS Nano 2013, 7 , 10263-10270. 
50. Kale, M. J.; Avanesian, T.; Xin, H.; Yan, J.; Christopher, P. Controlling Catalytic Selectivity on Metal Nanoparticles by Direct Photoexcitation of Adsorbate-Metal Bonds. Nano Lett. 2014, 14, 5405-5412.

51. Kazuma, E.; Jung, J.; Ueba, H.; Trenary, M.; Kim, Y. Real-Space and Real-Time Observation of a Plasmon-Induced Chemical Reaction of a Single Molecule. Science 2018, $360,521-526$.

52. Hoggard, A.; Wang, L.-Y.; Ma, L.; Fang, Y.; You, G.; Olson, J.; Liu, Z.; Chang, W.S.; Ajayan, P. M.; Link, S. Using the Plasmon Linewidth To Calculate the Time and Efficiency of Electron Transfer between Gold Nanorods and Graphene. ACS Nano 2013, ๆ, 11209-11217.

53. Perdew, J. P.; Burke, K.; Ernzerhof, M. Generalized Gradient Approximation Made Simple. Phys. Rev. Lett. 1996, 7r7, 3865-3868.

54. Kuisma, M.; Ojanen, J.; Enkovaara, J.; Rantala, T. T. Kohn-Sham Potential with Discontinuity for Band Gap Materials. Phys. Rev. B 2010, 82, 115106.

55. Yan, J.; Jacobsen, K. W.; Thygesen, K. S. First-Principles Study of Surface Plasmons on $\operatorname{Ag}(111)$ and $\mathrm{H} / \operatorname{Ag}(111)$. Phys. Rev. B 2011, 84, 235430.

56. Rossi, T. P.; Lehtola, S.; Sakko, A.; Puska, M. J.; Nieminen, R. M. Nanoplasmonics Simulations at the Basis Set Limit through Completeness-Optimized, Local Numerical Basis Sets. J. Chem. Phys. 2015, 142, 094114. 


\section{Graphical TOC Entry}

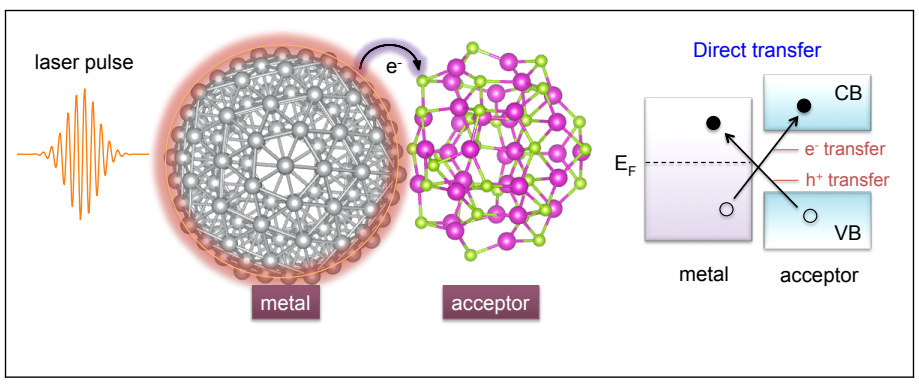

